\title{
Celebrating an illustrious career in breast cancer research, SERMS and mentorship
}

\author{
V Craig Jordan $*, 1$ \\ ${ }^{1}$ Department of Breast Medical Oncology, University of Texas MD Anderson Cancer Center, Houston, TX 77030, USA \\ * Author for correspondence: VCJordan@mdanderson.org
}

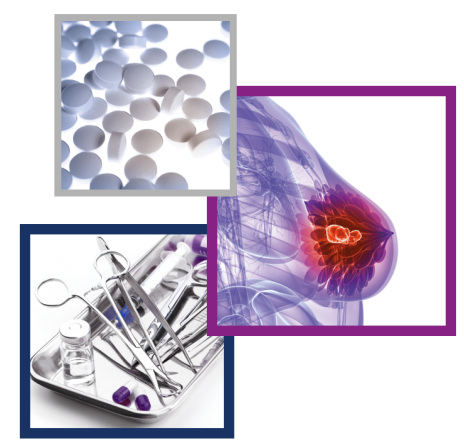

"My career was started by being in the right place at the right time, meeting the right people who could help me as mentors."

\begin{abstract}
V Craig Jordan speaks to Roshaine Wijayatunga, Managing Commissioning Editor: V Craig Jordan, OBE, PhD, DSc, MDhc, FMedSci, FAACR is Dallas/Ft Worth Living Legend Chair of Cancer Research, Professor of Breast Medical Oncology, Professor of Molecular and Cellular Oncology, and Chief, Section of Basic Science Research and Pharmacology. He trained as a pharmacologist at Leeds University Medical School and subsequently held Professorial appointments at the University of Wisconsin, Madison, Northwestern University, Chicago (Diana, Princess of Wales Professor of Cancer Research) and the Fox Chase Cancer Center, Philadelphia (the Alfred G Knudson Chair of Cancer Research). He was Professor of Oncology and Pharmacology at Georgetown University Medical Center and the Scientific Director of the Georgetown Lombardi Comprehensive Cancer Center, Washington, DC, USA. He developed the scientific principles for the effective use of tamoxifen, as an adjuvant therapy for breast cancer and the use of tamoxifen, as the first chemopreventive for breast cancer. His discovery and translational research of the novel group of medicines, known as Selected Estrogen Receptor Modulators, resulted in the development of raloxifene, for the prevention of osteoporosis and breast cancer. His contributions to medicine have been recognized with more than four dozen International Awards, including the Inaugural Brinker International Award (Susan G Komen for the Cure), David A Karnofsky Award (ASCO), the American Cancer Society (ACS) Medal of Honor, ACS Chemoprevention Prize (ASCO), the Charles F Kettering Prize and the St Gallen International Breast Cancer Prize. He is a Member of the National Academy of Sciences, a Fellow of the Academy of Medical Sciences and the National Academy of Medicine, and an Honorary Fellow of the Royal Society of Medicine. In 2002, he was appointed Officer of the Most Excellent Order of the British Empire, for services to International Breast Cancer Research.
\end{abstract}

First draft submitted: 7 August 2017; Accepted for publication: 14 November 2017; Published online: 21 December 2017

Keywords: fulvestrant $\bullet$ selective estrogen receptor disruptors $\bullet$ selective estrogen receptor modulators $\bullet$ tamoxifen

In an article that you published in Breast Cancer Management in 2015, you speak of how selected estrogen receptor modulators exploit the best target in oncology, namely, the estrogen receptor. What advances have you seen in this area in the past 2 years?

The selected estrogen receptor modulators (SERMs) have now been superseded by aromatase inhibitors (AI) for the long-term adjuvant therapy for postmenopausal patients with estrogen receptor (ER)-positive breast cancer. The AI have an advantage over the SERM tamoxifen in having a decrease in recurrence rates, improved decreases in contralateral breast cancer and fewer side effects such as endometrial cancer, and thromboembolic disorders. During the past 5 years, the research community has discovered that patients receiving AI therapy have an increased incidence of mutations in the ER. The incidence of mutations is about $20 \%$. Patients receiving adjuvant tamoxifen do not have similar mutations in the ER. The significance of the mutations in the ER for patients formally receiving $\mathrm{AI}$ is that the ER is autostimulating for tumor growth without the necessity of estrogen binding to the receptor. Additionally, second-line therapies are necessary to treat recurrent breast cancer that has a mutant ER. Current therapies are weakly active. The past 2 years have seen a number of new orally active pure antiestrogens that bind 
to the ER and destroy it. The first selective ER destroyer fulvestrant is not orally active and is weakly active at the mutant ER.

Although an increased investigation of orally active selective estrogen receptor disruptors (SERDs) is a natural response by medicinal chemists in the pharmaceutical industry, it may be time to rethink the whole strategy of longterm adjuvant therapy for breast cancer. It is essential that patients want to take long-term therapy for additional health benefits. Side effects from pure antiestrogens reduce persistence in taking the medicines during long-term adjuvant therapy resulting in recurrence and death. We understand the structure function relationships necessary to destroy the tumor ER and we know that this can be inserted into a SERM. The ideal agent for long-term adjuvant therapy would be an SERM that prevents osteoporosis, reduces coronary heart disease, prevents breast and endometrial cancer, but destroys the ER. All of this are possible and would aid patients through long-term recurrence-free survival.

\section{It was recently announced that you have been elected to the National Academy of Medicine for your work on SERMs. Congratulations for this great achievement. What does this honor mean to you?}

I was thrilled to receive this surprise email that my election was soon to be announced. I was unaware that I had been nominated. First, I am grateful to those who nominated me, but for me this is a distinction for the members of my Tamoxifen teams that have turned ideas into lives saved. We started all of our work in the 1970 s to convert a failed contraceptive ICI 46,474 into a medicine to treat breast cancer. We came out with the principles of targeting the tumor ER, use long-term or indefinite adjuvant tamoxifen therapy and we demonstrated that we can prevent tumor formation in animals - chemoprevention was possible. When I was recruited to Wisconsin to head their breast cancer program and start a new Tamoxifen team there; I was yet unaware that tamoxifen would be the pioneering SERM. It was at the Wisconsin Comprehensive Cancer Center that my team tested a potential competitor for tamoxifen for the treatment of breast cancer. The competitor failed in our animal test and tamoxifen was always the winner, except we discovered that tamoxifen had the potential to increase the growth of endometrial cancer and this would be a dangerous side effect for long-term tamoxifen therapy. The competitor did not increase endometrial cancer growth in the laboratory. By coincidence, I was asked to look at the impact of tamoxifen on animal bone density. The standard at the time to prevent osteoporosis was estrogen, so it was reasoned that antiestrogens like tamoxifen, and also the competitor, would make osteoporosis worse. We were surprised to find, in the mid-1980s, that tamoxifen and the competitor now called raloxifene were better at preserving bone density than estrogen alone. My Wisconsin Tamoxifen team had discovered a principle: an SERM, wisely chosen for safety, could prevent the growth of breast and endometrial cancer but have an estrogenic tickle to prevent osteoporosis and coronary heart disease. There are now five US FDA-approved SERMs, all with chemical connection to my Wisconsin Tamoxifen team. To me, election to the National Academy of Medicine recognizes the contribution of my Tamoxifen teams to advance women's health. On a personal level, I take great pride in the opportunities seized by my team members who have advanced in their careers to become chairs of departments, research directors or Vice Presidents of pharmaceutical companies and one who has become the Director of her nation's National Cancer Research Center.

You recently authored a paper on the topic of, "Endoxifen: The end, or are we at the beginning?" in the Journal of Clinical Oncology. What issues did you explore in this feature? Endoxifen is an important antiestrogenic metabolite of tamoxifen with a high affinity to the tumor ER. It has been hypothesized that the metabolite is essential for tamoxifen action as aberrations in the CYP2D6 gene were associated in the literature with lower tamoxifen activity as a breast cancer medicine. This hypothesis was never proven in prospective clinical trials and genotyping is not standard of care. My editorial in the Journal of Clinical Oncology commented on a Phase I clinical trial from the Mayo group that examined increasing doses of endoxifen to treat antihormone-resistant breast cancer. Responses were around 30\%. However, I made the point that low-dose estrogen therapy is reported to produce the same response rates in the same category of patient. Nevertheless, other innovations for the use of added endoxifen to enrich adjuvant tamoxifen therapy are ongoing in Germany. So, the point I was emphasizing was that although tamoxifen is the first targeted therapy to save millions of lives in women with ER-positive breast cancer, the SERM saga may not be over and improvements can be made. 


\section{What are the recent developments in tamoxifen research?}

Tamoxifen has remained a reliable, safe medicine used throughout the world for the past 40 years. Recent research on the association of endometrial cancer and tamoxifen concluded that women without obvious endometrial pathologies have no increase in endometrial cancer with tamoxifen. Indeed, endometrial cancer was a very rare event. 30 years ago, the concerns about a link between endometrial cancer and tamoxifen were very different because gynecologists were not effectively involved with breast cancer patient care. It took the translational research from Wisconsin to insure changes in medical care [1].

\section{What are your thoughts on chemoprevention in breast cancer, in particular, nonadherence?}

The strategy as previously conceived to test the worth of tamoxifen to prevent primary breast cancer in high-risk women compared and contrasted control groups taking placebo and a tamoxifen group taking 5 years of tamoxifen or more. Significant decreases in breast cancer were found in tamoxifen-treated women in all major studies. However, there were two flaws in this design. First, women without disease passing through the menopausal years have similar side effects whether you are taking tamoxifen or not. This has been documented in recent post-trial analysis. So, side effects for women without disease are the most important factor to insure patients take a known and effective inhibitor of cancer. Second, the incidence of breast cancer is very small even in populations of women with high risk. In other terms, the majority of women will remain cancer-free, but all women will have fear of tamoxifen-related side effects. This leads to nonadherence. As a result, in 1990 [2], I suggested that SERMs should be found that were safe, but used to treat major diseases in postmenopausal women such as osteoporosis or coronary heart disease. The hypothesis to be tested was that if osteoporosis is treated with an SERM, then breast cancer would be prevented at the same time. We found this to be true [3]. This I think is the best strategy to address major diseases in women until the day arrives that we can tell exactly, by genetic testing, that a woman will develop spontaneous breast cancer at a certain point in her lifetime.

\section{You are celebrating a great milestone of a birthday this year! Congratulations \& happy birthday from all of us here at Breast Cancer Management journal. What would you say your long \& successful career is down to? What inspires you or drives you to keep up with your research day by day?}

I was a pharmacologist who wanted to develop drugs to treat cancer but this was unfashionable in the 1970s. My career was started by being in the right place at the right time, meeting the right people who could help me as mentors. At that time, very few people even considered that antiestrogen therapy was going to be a useful therapeutic strategy to treat breast cancer. Chemotherapy was king and the medicines killed cancer cells. As a result, this strategy was the primary strategy around the world. The breakthrough came with me using my pharmacology training to target the ER in tumors, but then seeking out influential mentors (Elwood Jensen, Bill McGuire) who could teach me laboratory techniques that I needed to develop my plan. Through a study of models and mechanisms, tamoxifen was developed as a medicine. The discovery was subsequently made clinically that long-term adjuvant therapy with tamoxifen would save lives.

My start in the 1970s with my Tamoxifen team in Leeds University, England, allowed me to create a plan that resulted in my recruitment by Paul Carbone and Harold Rush to develop a breast program at the Wisconsin Clinical Cancer Center (WI, USA). The investment in me by them gave medicine SERMs and then one thing led to another! But my strategy had a dark side. If I was advocating long-term adjuvant tamoxifen therapy, then we needed to know about acquired drug resistance to tamoxifen. Tamoxifen was believed to be only holding cancer growth, so it was not killing cancer. Eventually tamoxifen would fail. Marco Gottardis, an excellent student at Wisconsin was the first to discover that acquired resistance to tamoxifen occurred because the tumor cells grew with tamoxifen treatment, not despite tamoxifen treatment. To combat this, we tested at Wisconsin the first SERD that became fulvestrant in clinical practice today. Another PhD student at Wisconsin, Doug Wolf made the discovery that long-term tamoxifen therapy for up to 5 years creates a new form of drug resistance to antihormone therapy. Unexpectedly, low doses of estrogen killed the cancer cells and did not stimulate them to grow. This led to our extensive studies of estrogen-induced apoptosis and these ideas have been translated to successful clinical trials by others. The results that were unanticipated by our experiments actually have had the greatest impact in changing clinical practice. Throughout I have maintained my publishing record as a molecular pharmacologist for ligands at the ER. My original PhD in "Failed contraceptives" (1969-1972) was to crystalize the purified ER with an estrogen and an antiestrogen. I rapidly found that this was not possible with the technology at the time, but this 
was accomplished 25 years later by others! Currently we are revisiting my PhD to decipher how different estrogens regulate estrogen-induced apoptosis by changing and modulating the ER complex. I hope to complete my $\mathrm{PhD}$ before I retire.

\section{As an established scientist with many years of experience under your belt so to speak, how important is it to you, to take time out of your schedule to mentor newer researchers \& oncologists as they begin \& progress through their career?}

For me, it is not just about the goal of research, but the lives that can be transformed given opportunities to excel. In my Tamoxifen teams over 40 years, it is the young trainees who took our ideas and turned it into lives saved through their experiments. We have a motto "WE ARE IN IT FOR LIFE". Our work continues to save lives, but I will mentor and advise my team members for the rest of their lives. Balkees Abderrahman is a case in point. She visited MD Anderson 2 years ago following her MD at medical school in Jordan. It was clear from the start she possessed natural talents in writing and focused career objectives. After 2 years of diligent work, she has an extensive CV and bibliography with contributions to editorials, an obituary in Nature, two contributions to major breast cancer medical reference books and numerous social commentaries in, for example, The Hill and Huffington Post. She has spoken at a youth conference at the UN and her commentary on gender equality was placed on the UN website for International women's day. In mentoring, my goal is to encourage independent achievement. She is now a special correspondent for the Pharmaceutical Journal in the UK and is working in a similar role with Nature. In the laboratory, she is up to speed with all techniques and she has earned authorship on two refereed publications. Her independent study mentored by Dr Nuhad Ibrahim member in our department, earned her a scholarship to attend the European Society for Medical Oncology (ESMO) in Madrid this year to participate in a Poster Discussion session. Such is her positive response to opportunities given, she will soon begin a split site $\mathrm{PhD}$ between Leeds University and MD Anderson. This is specifically for candidates of high quality. I got my degrees all from Leeds, so this continues that bond.

Balkees is not alone. Former members of my Tamoxifen teams over 40 years became Chairs of departments, Associate Deans, Leaders in Industry (Research Directors or Vice Presidents) and one became the Director of her nation's Cancer Research Institute. My mentors gave me opportunities and I seized them with alacrity. Now, it is my turn to create a future for young talented people in medical science, so they can build on success and save further lives.

\section{What would you say are your career highlights to date? What would you list as your greatest achievements?}

I think I have been very fortunate to have been talent spotted by some of the great leaders in cancer research who gave an opportunity to a young man with a big idea: develop a completely different treatment strategy for treating breast cancer. I was fortunate to have been at the beginning of the reinvention of ICI 46,474 to become tamoxifen and to have been invited to compare and contrast tamoxifen with a failed breast cancer drug that became raloxifen. My Tamoxifen teams of enthusiastic young investigators were the individuals that turned ideas into lives saved. The community of scholars at the Worcester Foundation, Leeds University, the Wisconsin Clinical Cancer Centre and the Robert H Lurie Comprehensive Cancer Centre in Chicago (then Director Steven T Rosen) were the perfect places to make new ideas come to fruition. For me, it has always been about team work and creating careers for others with the goal of only focusing on research that results in patients surviving from a diagnosis of breast cancer. This was hopeless as a goal in the 1970s when my career started. But the success of tamoxifen and the discovery of SERMs revolutionized women's health. I feel privileged to have witnessed, during my career, our laboratory translational research be replicated in the clinical trials that created improvements in women's health. There are now five FDA-approved SERMs for different indications in women's health that have their origins on basic research from my laboratory. As personal career highlights, who would imagine that I would become the Diana Princess of Wales Professor of Cancer Research, Her Majesty Queen Elizabeth II would appointment me to the Order of the British Empire, or design, equip and staff a Ludwig Institute for Cancer Research in Bern, Switzerland, during an exciting year in 1979. 


\section{How do you see the field of breast cancer management changing in the near future? What needs to change?}

We now know that long-term antiestrogenic adjuvant therapy saves lives. However, research cannot stand still, and the question must be asked 'how can this advance be improved?' My goal by accepting the invitation to join the staff at the University of Texas MD Anderson Cancer is to do just that. We have new technologies to identify patients at high risk of relapse following surgery and we have new precision medicines to kill antihormone-resistant breast cancer. My plan is to focus on high-risk patients and use preemptive cocktails of precision medicines to destroy micrometastatic breast cancer [4]. We propose annual drug holidays as recently tested in the Study Of Letrozole Extension. If there is one thing we have learned about tamoxifen it is that administered for metastatic breast cancer at the end of life everybody dies. By contrast, the administration of tamoxifen to patients as an adjuvant to control micrometastatic disease, dramatically improves patient survival - we build on that!

\section{Disclaimer}

The opinions expressed in this interview are those of the interviewee and do not necessarily reflect the views of Future Medicine Ltd.

\section{Financial \& competing interests disclosure}

This article was funded by a National Institutes of Health MD Anderson's Cancer Center Support Grant, CA016672. The author has no other relevant affiliations or financial involvement with any organization or entity with a financial interest in or financial conflict with the subject matter or materials discussed in the manuscript apart from those disclosed.

No writing assistance was utilized in the production of this manuscript.

\section{References}

1 Potkul RK, Unger JM, Livingston RB et al. Randomized trial of medroxyprogesterone acetate for the prevention of endometrial pathology from adjuvant tamoxifen for breast cancer: SWOG S9630. NPJ Breast Cancer 2, 16024 (2016).

2 Lerner LJ, Jordan VC. Development of antiestrogens and their use in breast cancer: eighth Cain memorial award lecture. Cancer Res. 50(14), 4177-4189 (1990).

3 Cummings SR, Eckert S, Krueger KA et al. The effect of raloxifene on risk of breast cancer in postmenopausal women: results from the MORE randomized trial (Multiple Outcomes of Raloxifene Evaluation). JAMA 281(23), 2189-2197 (1999).

4 Abderrahman B, Jordan VC. Rethinking extended adjuvant antiestrogen therapy to increase survivorship in breast cancer. JAMA Oncology. Published online Nov 16 (2017). 